\title{
FLÚOR NA ÁGUA DO AQUÍFERO BAMBUÍ NO OESTE DA BAHIA (BRASIL)
}

\author{
GONÇALVES, M.V.P. ${ }^{1 *}$; CRUZ, M.J.M.'; SANTOS, R.A. ${ }^{1}$; RAMOS JUNIOR, A.B.S. ${ }^{1}$; COUTINHO, \\ C.A.M. ${ }^{1}$
}

1. Grupo de Geoquímica das Interfaces - Universidade Federal da Bahia.

*Corresponding author: zoovitor81@yahoo.com.br

\begin{abstract}
Gonçalves, M.V.P. ${ }^{1 *}$; Cruz, M.J.M. ${ }^{1}$; Santos, R.A. ${ }^{1}$; Ramos Junior, A.B.S. ${ }^{1}$; Coutinho, C.A.M. ${ }^{1}$ (2018). Assesment of Presidente Getúlio waterfalls bathing conditions - Santa Catarina State, Brazil. Braz. J. Aquat. Sci. Technol. 22(1). eISSN 1983-9057. DOI: 9654/bjast.v22n1. This paper reports the scientific investigation basic hydrogeochemistry of the Bambuí Aquifer in western Bahia, emphasizing on fluorine. Water was sampled from 61 wells during the years of 2011 (rainy season) and 2012 (dry season). The $\mathrm{pH}$ ranged between 6.87 and 8.93 , with predominantly alkaline conditions. The calcic bicarbonated hydrochemical facies (56\%), calcic mixed facies $(20 \%)$ and sodic bicarbonated facies $(10 \%)$ were the most representative in this region, in which fluoride concentrations exceeded the local optimal limit of fluoride $(0.8$ mg.L-1) for $25 \%$ of the wells, which varied between the range of 0.1 and $6.20 \mathrm{mg} . \mathrm{L}-1$ and the mean of $0.96 \mathrm{mg} . \mathrm{L}-1$. The Principal Components Analysis and composition of the water samples showed the relevance of chemical weathering for mineralization and groundwater quality; as well as for understanding fluoride enrichment. The origin of fluorine reflects the importance of the weathering of the rocks of the Bambui Aquifer and the dissolution of minerals hosted these for the quality of the water. It is recommended for the study site strong public investment in research in the field of geosciences and public health and desalination of brackish water. One should also prioritize the monitoring of groundwater quality, nitrate pollution and epidemiological surveillance of dental fluorosis or skeletal fluorosis related to ingestion of groundwater. The results of this research reveal the relevance of water resource management to overcome the challenges of basic sanitation and public health promotion. The results of this research show the relevance of water resources management to improve basic sanitation and promote public health.
\end{abstract}

Keywords: Groundwater, Fluorite, Fluoride, Geomedicine.

\section{INTRODUÇÃO}

A água subterrânea pode conter íons em teores adversos à saúde (Rebouças, 2006). A relação flúorsaúde tem relevância mundial, cuja ingestão de águas naturais ricas em flúor é a principal via de exposição, um fator de risco de fluorose dental e esquelética (Komati \& Figueiredo, 2013; Raju, 2017).

Krauskopf \& Bird (1995) estimaram um teor médio de flúor crustal entre 500 e $1000 \mathrm{mg} \cdot \mathrm{kg}^{-1}$. Nas rochas, o flúor apresenta ocorrência intimamente relacionada aos processos ígneos (Bell, 1998). Constitui, amiúde, a fase volátil, nas etapas finais da evolução de rochas alcalinas (Dardenne et al., 1997).

O flúor, litófilo, é o elemento de maior eletronegatividade. Possui um raio iônico similar ao da hidroxila $\left(\mathrm{OH}^{-}\right)$, por esta razão a substitui na diferenciação magmática. Segundo Naseem et al. (2010), o flúor penetra nos silicatos tardios, por conta do alto coeficiente de partição para minerais de baixa temperatura.

A fluorita $\left(\mathrm{CaF}^{2}\right)$ é o mineral de flúor mais comum na natureza (Naseem et al., 2010). Encontrado, igualmente, nos anfibólios, micas, fluorapatita, no topázio e criolita. Segundo Brindha et al. (2011), o intemperismo químico de minerais de flúor o libera para a atmosfera, solos, poeiras, biota e às águas.

O flúor nas águas naturais ocorre em especial, desde teores traço até $2.800 \mathrm{mg} \cdot \mathrm{L}^{-1}$ (Apambire et al., 1997). A Portaria 2914/11 (BRASIL, 2011), e WHO (2006), preconizam um limite de $1,5 \mathrm{mg} \cdot \mathrm{L}^{-1}$ na água potável. Este deve ser ajustado em função da temperatura média do ar (Mendes \& Oliveira, 2004).

Soares et al. (2012) esclarecem que o uso de doses ótimas do flúor em odontologia contribui à saúde bucal, embora a fluorose dentária correlaciona-se com a ingestão de doses tóxicas do flúor na formação do esmalte dental. A fluorose dentária é uma hipomineralização do esmalte dental, em que as alterações na constituição, manchas ou perda da estrutura do esmalte, podem vir a refletir problemas estéticos, funcionais e psicológicos (Rigo et al., 2014).

Gallará et al. (2011) orientam que a ingestão de água com teor ótimo de flúor pode prevenir tanto a cárie quanto a fluorose dentária. Entretanto, a exposição a doses tóxicas ou deficitárias representa risco de cárie ou fluorose. O efeito adverso do flúor à saúde deve-se a dose de exposição (Parreiras et al., 2009). Segundo Fujibayashi et al. (2011), a fluorose atinge crianças com até seis anos de idade, ocasião em que o esmalte dentário está em formação.

A prevalência da fluorose dentária e óssea endêmica abrange 200 milhões de pessoas, especialmente na Índia e na China (Vikas et al., 2013). No Brasil, pesquisas pautaram a relação flúor na água 
subterrânea e a fluorose (Licht et al., 1996; Cangussu et al., 2004; Toassi \& Abegg, 2005; Velásquez et al., 2006; Ferreira et al., 2010; Komati \& Figueiredo, 2013; Cruz et al., 2015).

Velásquez et al. (2006), Costa et al. (2013), Cruz et al. (2015) estudaram a relação flúor-saúde no Aquífero Bambuí, usada para o consumo humano, no oeste da Bahia e norte de Minas Gerais, com clima de subúmido a semiárido. A prevalência da fluorose dentária, em escolares aos 12 anos de idade, em São Francisco (MG) foi de $75-100 \%$, e a severidade de $46-82 \%$ (moderada/grave). Em Santana (BA), variou de $26-100 \%$, e a severidade de $25-100 \%$ (moderada/ grave).

No Aquífero Bambuí, no oeste da Bahia (Figura 1), destacam-se, entre os estudos anteriores, as pesquisas hidroquímicas realizadas pela CODEVASF (1989), Negrão (2007), Camurugy (2009), Coutinho (2014) e Gonçalves (2014). Este aquífero cárstico carece de estudos hidrogeoquímicos aplicáveis à gestão da qualidade da água. Para tanto, este artigo almeja investigar a hidrogeoquímica básica do Aquífero Bambuí no oeste da Bahia, com especial ênfase no flúor.

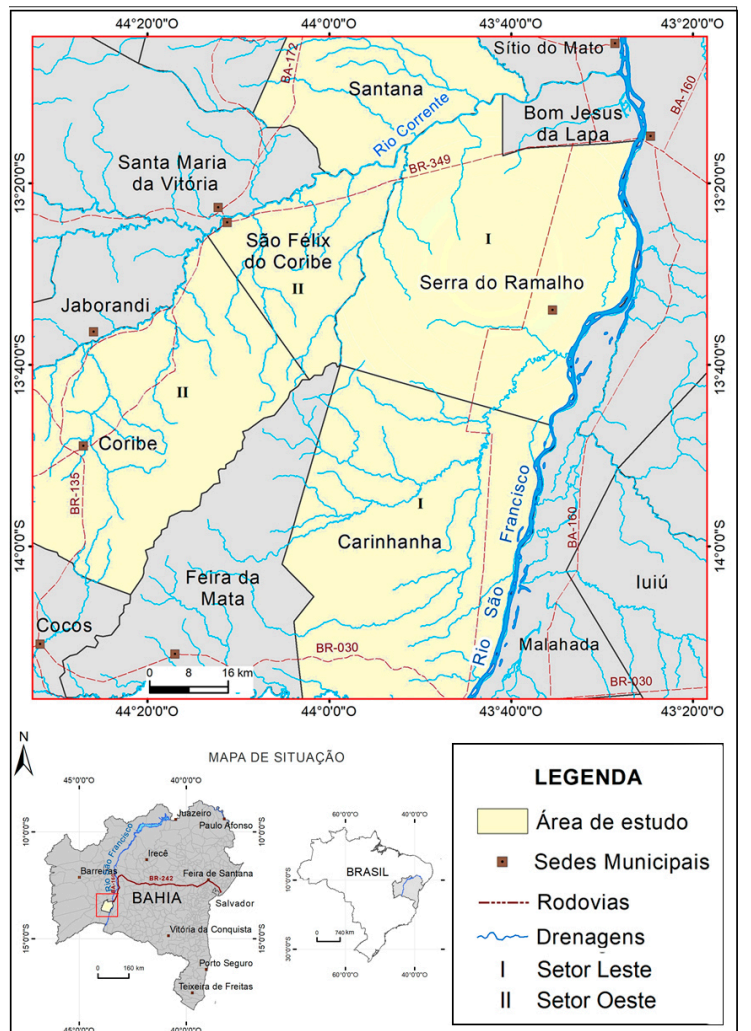

Figura 1 - Mapa de localização e situação do sítio de estudo, da Bahia.

\section{MATERIAIS E MÉTODOS}

\section{Área de estudo}

O sítio de estudo tem área de $10622 \mathrm{~km}^{2}$ e habitantes, 63.053 urbanos e 49.070 rurais, e Produto Interno Bruto (PIB) de $\mathrm{R} \$ 569.253,00$ reais (IBGE, 2010). O Índice de Desenvolvimento Humano Municipal (IDHM) varia de 0,6 a 0,68 (PNUD, 2010).

O clima varia de subúmido a semiárido, com temperatura média anual de $26,1^{\circ} \mathrm{C}$, precipitação de $949 \mathrm{~mm} / \mathrm{ano}$, concentrada de novembro a abril, e estiagem de maio a setembro (INMET, 2013), segundo dados das estações meteorológicas de Bom Jesus da Lapa, Correntina e de Carinhanha, na Bahia.

$\mathrm{Na}$ geologia regional afloram as rochas neoproterozóicas pelito-carbonáticas do Grupo Bambuí, depositadas sobre o embasamento cristalino, gnáissico-migmatítico, de idade Arqueano-Paleoproterozóico (Misi et al., 2011). Destacam-se calcários e dolomitos da Fm. Sete Lagoas, base do Grupo Bambuí, que hospedam depósitos de fluorita (Misi et al., 2000). As coberturas detríticas ou aluviais do limite entre o Terciário e Quaternária e arenitos do Cretáceo do Grupo Urucuia têm relevância regional. Os poços perfurados nestas rochas, no contexto do aquífero Urucuia, contribuem ao abastecimento, em especial nas áreas rurais.

No oeste da Bahia, o Aquífero Urucuia deposita-se sobre Aquífero Bambuí, e contribui em escala regional para a recarga do Aquífero Bambuí, junto às chuvas locais (Negrão, 2007). A Figura 2 mostra a direção principal do fluxo subterrâneo, que se move de Oeste para Leste, para o rio São Francisco, o nível de base.

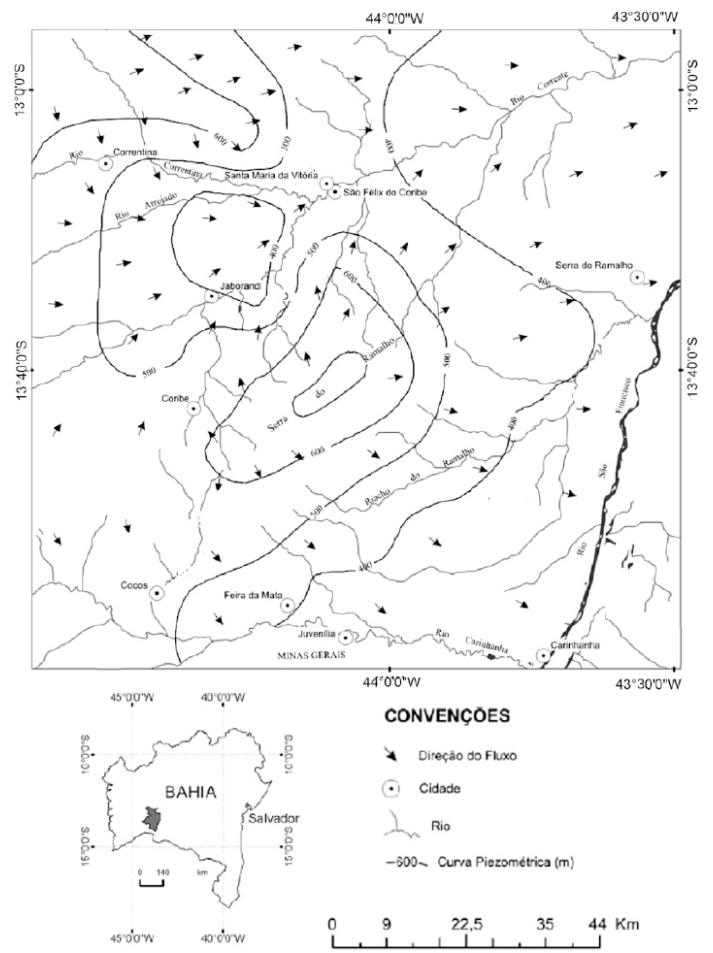

Figura 2 - Mapa das superfícies potenciométrica do sítio de estudo. Fonte: Modificado de Camurugy (2009). 


\section{Análises laboratoriais}

Foram coletadas amostras de água em 61 poços tubulares em novembro de 2011 (período chuvoso) e julho de 2012 (estiagem) (Tabelas 1-2), e tomadas medidas de variáveis físico-químicas $(\mathrm{pH}$, ORP, CE, STD) in situ, por sonda multiparâmetros, bem como alíquotas para as análises laboratoriais.

A leitura de cátions $\left(\mathrm{Na}^{+}, \mathrm{K}^{+}, \mathrm{Ca}^{2+}, \mathrm{Mg}^{2+}\right)$ deu-se por Espectrometria de Emissão Óptica com Plasma Indutivamente Acoplado (ICP-OES), em duplicata, sendo $20 \%$ em triplicatas. Os ânions foram mensurados por titrimetria $\left(\mathrm{HCO}_{3-}, \mathrm{Cl}\right)$, espectrofotometria $\left(\mathrm{SO}_{4}{ }^{2-}, \mathrm{NO}_{3}\right)$ e por meio da colorimetria (SPANSD) (F-).

A estatística abrangeu testes de normalidade (Shapiro-Wilk), comparações múltiplas para dados paramétricos (ANOVA), ou não-paramétricos (Kruskal-Wallis), com significância de 95\%, e a Análise das Componentes Principais (PCA). Também, pelo tratamento de dados hidroquímicos de 217 poços do Sistema de Informações de Águas Subterrâneas (SIAGAS), do Serviço Geológico do Brasil.

\section{Resultados}

As Tabelas 1 e 2 apresentam os resultados hidroquímicos. Os valores do $\mathrm{pH}$ foram de 6,9 a 8,9, com distribuição gaussiana em $2011(p=0,31)$, sendo similares nos períodos amostrais. Predominam condições oxidantes em $82 \%$ dos poços, com valores da ORP de -181 a $362 \mathrm{mV}$, distribuição não gaussiana $(p=0,04 ; p<0,01)$, cujas medianas não diferem entre 2011-2012 $(p=0,79)$.

Os valores da condutividade elétrica (CE) variaram de 286 a $2.165 \mu \mathrm{S} . \mathrm{cm}^{-1}$, com distribuição não gaussiana $(p<0,01)$, cujas medianas não diferem entre as amostras $(p=0,79)$ (Tabelas 1-2). Os sólidos totais dissolvidos (STD) foram de 186 a $1.407 \mathrm{mg} \cdot \mathrm{L}^{-1}$, e as medianas não diferem entre 2011 e $2012(p=0,66)$. A dureza total variou de 25 a $682,4 \mathrm{mg} . \mathrm{L}^{-1}$, e as médias não diferem nas amostras $(p=0,56)$.

Tabela 1 -Resultados das análises hidroquímicas das amostras de água subterrânea coletada em dezembrode 2011, período chuvoso, no oeste da Bahia.

\begin{tabular}{|c|c|c|c|c|c|c|c|c|c|c|c|c|c|c|}
\hline \multirow{2}{*}{ Parâmetro } & \multirow{2}{*}{\multicolumn{2}{|c|}{$\begin{array}{l}\mathrm{mV} \\
\text { ORP }\end{array}$}} & \multirow{2}{*}{$\begin{array}{c}\mu \mathrm{SS} . \mathrm{cm}^{-1} \\
\mathrm{CE}\end{array}$} & \multicolumn{11}{|c|}{$\mathrm{mg} \cdot \mathrm{L}^{-1}$} \\
\hline & & & & STD & $\mathrm{Na}^{+}$ & $\mathrm{K}^{+}$ & $\mathrm{Ca}^{2+}$ & $\mathrm{Mg}^{2+}$ & $\mathrm{Cl}$ & $\mathrm{HCO}_{3}$ & $\mathrm{SO}_{4}{ }^{2-}$ & $\mathrm{NO}_{3}$. & $\mathrm{F}$ - & DT \\
\hline Minimo & 6.9 & -181.0 & 286.0 & 186.0 & 1.9 & 0.4 & 5.9 & 1.2 & 12.1 & 82.4 & 8.1 & 0.0 & 0.1 & 25.0 \\
\hline Máximo & 8.7 & 362.0 & 2165.0 & 1407.3 & 245.4 & 5.3 & 220.5 & 40.7 & 460.0 & 427.1 & 236.2 & 15.0 & 5.8 & 682.4 \\
\hline Média & 7.7 & 114.2 & 896.3 & 580.0 & 41.3 & 2.3 & 102.7 & 15.4 & 95.1 & 268.3 & 45.7 & 3.2 & 0.9 & 320.0 \\
\hline Mediana & 7.7 & 138.0 & 830.9 & 532.0 & 19.5 & 2.1 & 104.7 & 14.1 & 78.4 & 275.8 & 34.0 & 2.1 & 0.5 & 337.6 \\
\hline DP & 0.4 & 116.1 & 332.9 & 214.8 & 54.8 & 1.2 & 51.8 & 8.0 & 81.1 & 62.0 & 38.8 & 3.6 & 1.2 & 147.1 \\
\hline Erro Padrão & 0.1 & 14.9 & 42.6 & 27.5 & 7.0 & 0.2 & 6.6 & 1.0 & 10.4 & 7.9 & 5.0 & 0.5 & 0.2 & 18.8 \\
\hline CV (\%)* & 5.7 & 101.7 & 37.1 & 37.0 & 132.8 & 51.0 & 50.5 & 52.3 & 85.3 & 23.1 & 84.9 & 110.2 & 133.1 & 46.0 \\
\hline Shapiro-Wilk** & 0.31 & 0.04 & $<0.001$ & $<0.001$ & $<0.001$ & 0.001 & 0.08 & 0.05 & $<0.001$ & 0.12 & $<0.001$ & $<0.001$ & $<0.001$ & 0.09 \\
\hline $\begin{array}{l}\text { Portaria 2.914/11 } \\
\text { ou OMS (2003) }\end{array}$ & $6.5-9.0$ & - & - & 1000.0 & 200.0 & - & 75.0 & 50.0 & 250.0 & - & 250.0 & 10.0 & 0.80 & 500.0 \\
\hline
\end{tabular}

* DP: Desvio padrão; **CV: Coeficiente de Variação; *** Teste de normalidade de Shapiro-Wilk.

Tabela 2 -Resultados das análises hidroquímicas das amostras de água subterrânea coletada em julho 2012, período de estiagem, no oeste da Bahia.

\begin{tabular}{|c|c|c|c|c|c|c|c|c|c|c|c|c|c|c|}
\hline \multirow{2}{*}{ Parâmetro } & \multirow{2}{*}{\multicolumn{2}{|c|}{$\begin{array}{l}\mathrm{mV} \\
\text { ORP }\end{array}$}} & \multirow{2}{*}{$\begin{array}{c}\mu \mathrm{S} . \mathrm{cm}^{-1} \\
\mathrm{CE}\end{array}$} & \multicolumn{11}{|c|}{$\mathrm{mg} \cdot \mathrm{L}^{-1}$} \\
\hline & & & & STD & $\mathrm{Na}^{+}$ & $\mathrm{K}^{+}$ & $\mathrm{Ca}^{2+}$ & $\mathrm{Mg}^{2+}$ & $\mathrm{Cl}$ & $\mathrm{HCO}_{3}$ & $\mathrm{SO}^{2}$ & $\mathrm{NO}_{3}$ & F- & DT \\
\hline Minimo & 6.9 & -181 & 292.0 & 190.0 & 2.1 & 0.3 & 5.5 & 1.2 & 17.6 & 75.2 & 9.8 & 0.0 & 0.1 & 26.4 \\
\hline Máximo & 8.9 & 238.0 & 1920.0 & 1230.0 & 238.3 & 5.3 & 197.3 & 34.8 & 578.5 & 366.0 & 172.2 & 16.4 & 6.2 & 634.2 \\
\hline Média & 7.7 & 83.9 & 869.8 & 558.8 & 39.8 & 2.1 & 97.5 & 14.8 & 103.5 & 216.1 & 47.6 & 2.6 & 0.9 & 304.9 \\
\hline Mediana & 7.6 & 117.0 & 826.0 & 526.5 & 20.3 & 1.9 & 101.9 & 14.4 & 85.1 & 215.2 & 41.0 & 1.1 & 0.5 & 314.7 \\
\hline $\mathrm{DP}$ & 0.5 & 103.8 & 290.7 & 185.5 & 50.9 & 1.2 & 48.5 & 7.2 & 88.8 & 52.5 & 35.3 & 3.9 & 1.3 & 137.2 \\
\hline Erro Padrão & 0.1 & 13.3 & 37.2 & 23.7 & 6.5 & 0.2 & 6.2 & 0.9 & 11.4 & 6.7 & 4.5 & 0.5 & 0.2 & 17.6 \\
\hline CV $(\%)^{*}$ & 6.6 & 123.7 & 33.4 & 33.2 & 127.9 & 56 & 49.8 & 48.4 & 85.8 & 24.3 & 74.0 & 149.3 & 134 & 45.0 \\
\hline Shapiro-Wik** & 0.01 & $<0.001$ & $<0.001$ & $<0.001$ & $<0.001$ & $<0.001$ & 0.13 & 0.04 & $<0.001$ & 0.55 & $<0.001$ & $<0.001$ & $<0.001$ & 0.39 \\
\hline $\begin{array}{l}\text { Portaria 2.914/11 } \\
\text { ou OMS (2003) }\end{array}$ & $6.5-9$ & - & - & 1000.0 & 200.0 & - & 75.0 & 50.0 & 250.0 & - & 250.0 & 10.0 & 0.80 & 500.0 \\
\hline
\end{tabular}

* DP: Desvio padrão; *CV: Coeficiente de Variação; ${ }^{\star * *}$ Teste de normalidade de Shapiro-Wilk 
Os teores dos cátions e ânions foram, em ordem decrescente: $\mathrm{rCa}^{2+}>\mathrm{rNa}^{+}>\mathrm{rMg}^{+}>\mathrm{rK}+\mathrm{e}$ $\mathrm{rHCO}_{3-}>\mathrm{rCl}^{-}>\mathrm{rSO}_{4}{ }^{2-}>\mathrm{rF}^{-}>\mathrm{rNO}_{3-}$ (Tabelas 1-2). O cálcio variou de 5,5 a $220,5 \mathrm{mg}^{-1} \mathrm{~L}^{-1}$, distribuição gaussiana $(p=0,08 ; p=0,13)$, cujas médias não diferem significativamente entre si nas amostras de 2011-2012 $(p=0,57)$. Os valores do sódio foram de 1,9 a 245 mg. $L^{-1}$, distribuição não gaussiana $(p<0,01)$, em que as medianas não diferem entre períodos de estiagem e chuvoso $(p=0,93)$.

Os teores do magnésio variaram de 1,2 e 40,7 mg.L-1 , com distribuição gaussiana em $2011(p=0,05)$ (Tabelas 1-2), cujas medianas não diferem nas amostras $(p=0,9)$. Oscilaram no potássio de 0,3 a 5,3 $\mathrm{mg} \cdot \mathrm{L}^{-1}$, com distribuição não normal $(p=0,001 ; p<0,01)$, cujas medianas não diferem entre 2011-2012 $(p=0,33)$.

Os valores do bicarbonato foram de 75,2 e 427,1 $\mathrm{mg} . \mathrm{L}^{-1}$, com distribuição gaussiana $(p=0,12 ; p=0,6)$ (Tabelas 1-2), cujas médias das amostras diferem entre si $(p<0,01)$. No cloreto, oscilaram de 12,1 a 578,5 mg. $L^{-1}$, com distribuição não gaussiana $(p<0,001)$, em que as medianas não diferem entre 2011-2012 $(p=0,3)$. Ocorrem níveis extremos e outliers, e superior ao limite de potabilidade (Figura 4).

As concentrações do sulfato foram de 8,1 a $236,2 \mathrm{mg} \cdot \mathrm{L}^{-1}$, distribuição não gaussiana $(p<0,01)$, cujas medianas não diferem nas amostras $(p=0,6)$. 0 nitrato variou de 0,01 a $16,4 \mathrm{mg} \cdot \mathrm{L}^{-1}$, distribuição não gaussiana $(p<0,01)$, cujas medianas diferem sazonalmente ( $p=0,03)$. O fluoreto oscilou de 0,1 a 6,2 mg.L-1, distribuição não gaussiana $(p<0,01)$, e as medianas não diferiram nas amostras $(p=0,8)$.

Destacaram-se as fácies bicarbonatadas cálcicas $(56 \%)$, mistas cálcicas $(20 \%)$ e fácies cloretadas cálcicas (10\%) (Figura 3), sendo que a incerteza do balanço iônico, baseado no erro prático, definido por Logan (1965), inferior a 10\%.

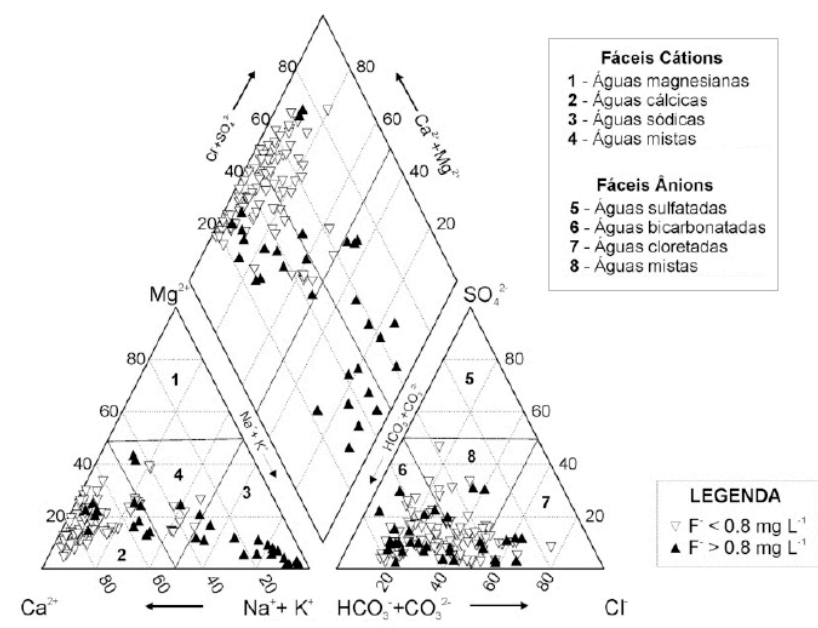

Figura 3 - Diagrama de Piper (1944), para a classificação das águas subterrâneas.

\section{DISCUSSÃO}

Excederam os limites de potabilidade preconizados pela WHO (2006), ou pela Portaria 2.914/11 (BRASIL, 2011), as variáveis cálcio (71\%), STD $(5 \%)$, DT $(7 \%)$, sódio $(2,5 \%)$, cloreto $(8 \%)$, fluoreto $(25 \%)$ e nitrato (8\%) (Tabelas 1-2). Deve-se ter atenção a mais $7 \%$ dos poços com teores do nitrato foram de 5 a 10 mg. $\mathrm{L}^{-1}$, porque este intervalo já indica a presença de fontes de poluição do corpo hídrico.

Os valores do $\mathrm{pH}$ revelaram águas alcalinas (96\%), com variabilidade similar entre as amostras e os dados do Sistema de Informações de Águas Subterrâneas (SIAGAS) (Figura 4). Os altos níveis do cálcio, similares nos dados de campo e do SIAGAS, refletem a interação água-rocha no Aquífero Bambuí.

A Portaria 2.914/11 não preconiza limite ótimo para o cálcio, cujo excesso influencia a dureza da água, dificulta a lavagem e o cozimento de alimentos e obstrui tubulações. O intemperismo das rochas do Aquífero Bambuí libera os íons $\mathrm{Ca}^{2+}$ e $\mathrm{Mg}^{2+}$ dos minerais para a água, em função do tempo de residência, pressão do $\mathrm{CO}^{2}$, temperatura e da presença de ácidos inorgânicos e orgânicos.

Moral et al. (2008) propõem que a hidroquímica de aquíferos cársticos reflete, especialmente, a dissolução da calcita e da dolomita. Fairchlid et al. (2000) destacam, igualmente, a proporção entre tais minerais e do tempo de residência. A dissolução de rochas carbonáticas imprime forte dureza e mineralização da água subterrânea, em função da composição química e mineralógica daquelas, fatores geoquímicos, pluviometria e fluxo da água (White, 2002; Negrão, 2007).

Os teores do sódio mostraram variabilidade similar entre 2011 e 2012 e os dados do SIAGAS, havendo pontos extremos e outliers (Figura 4). A variabilidade do sódio decorre, possivelmente, da distribuição dos pelitos na geologia local e da ação de reações de troca de bases em unidades aquíferas pelito-carbonático.

A composição físico-química da água subterrânea depende da mobilidade e solubilidade de minerais no intemperismo químico (Drever, 1997). Os íons $\mathrm{Ca} 2+, \mathrm{Mg} 2+$ e $\mathrm{Na}+$ são móveis e facilmente disponibilizados nas águas naturais pelo intemperismo, embora o íon $\mathrm{K}+$ ocupe uma posição intermediária.

Os teores do potássio nas águas naturais são comumente inferiores aos do sódio, por conta do seu comportamento hidrogeoquímico (Hem, 1985; Lima, 2010). A liberação do íon $K+$ dos minerais à solução é mais difícil do que o $\mathrm{Na}+$, tendendo a ser reincorporado pelos produtos neoformados, embora se for solvido permanece comumente na solução, água subterrânea, enriquecendo-a. 


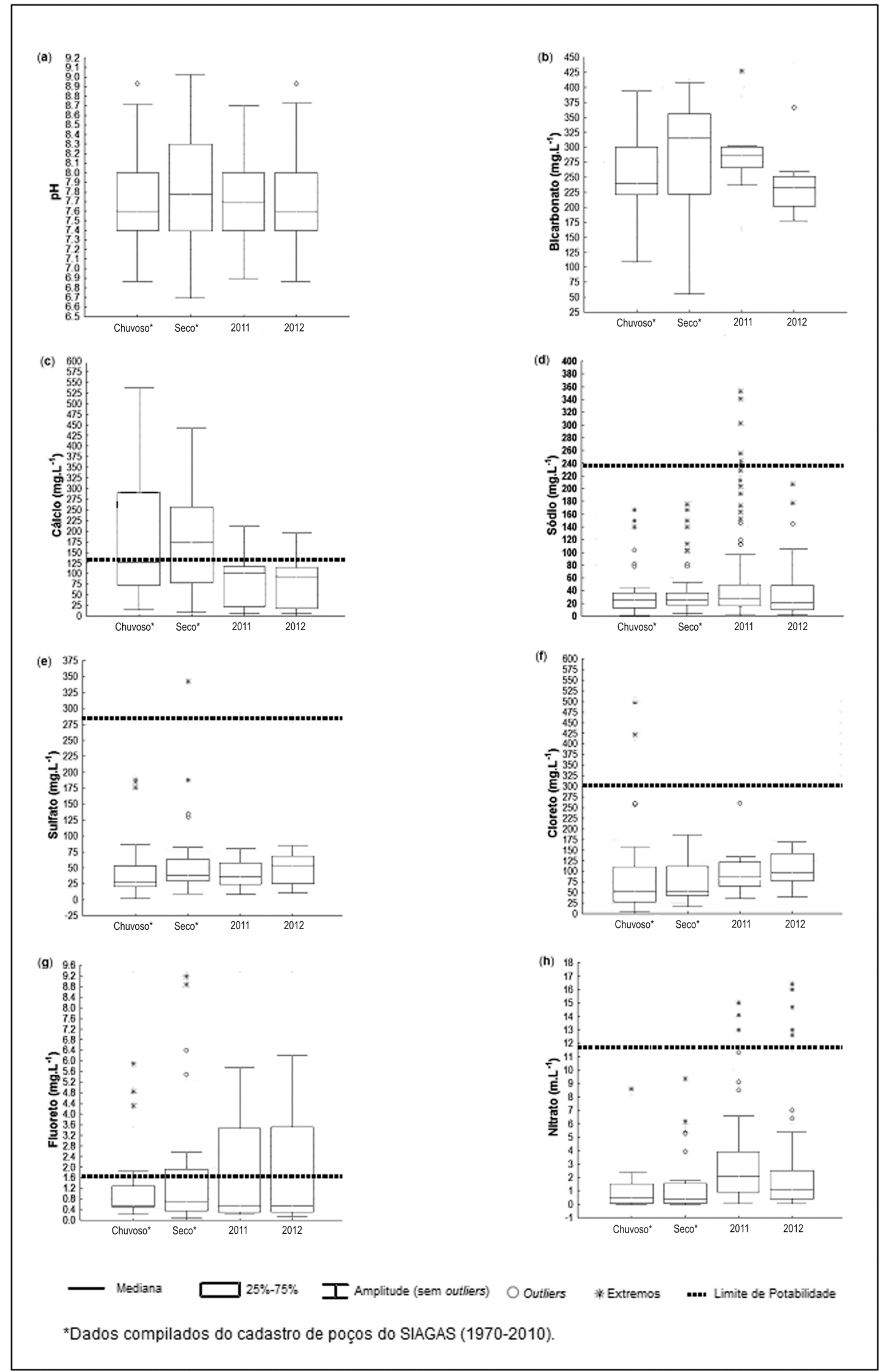

Figura 4 - Dispersão das variáveis físico-químicas das águas subterrâneas do sítio de estudo. 
A composição físico-química da água subterrânea depende da mobilidade e solubilidade de minerais no intemperismo químico (Drever, 1997). Os íons $\mathrm{Ca} 2+, \mathrm{Mg} 2+$ e $\mathrm{Na}+$ são móveis e facilmente disponibilizados nas águas naturais pelo intemperismo, embora o íon $\mathrm{K}+$ ocupe uma posição intermediária.

Os teores do potássio nas águas naturais são comumente inferiores aos do sódio, por conta do seu comportamento hidrogeoquímico (Hem, 1985; Lima, 2010). A liberação do íon $K+$ dos minerais à solução é mais difícil do que o $\mathrm{Na}+$, tendendo a ser reincorporado pelos produtos neoformados, embora se for solvido permanece comumente na solução, água subterrânea, enriquecendo-a.

Em meio cárstico, espera-se altos teores dos íons bicarbonato, como no Aquífero Bambuí, na Bahia, que decorre da dissolução de calcários e dolomitos do Grupo Bambuí, e da infiltração da água meteórica. Foi obtida menor mediana do íon bicarbonato em 2012, sem haver nítido padrão de dispersão (Figura 4).

O cloreto sofre influência da interação águarocha, circulação da água e clima semiárido, pela ação da evapotranspiração e irregularidade das chuvas, e constitui a hidroquímica das chuvas e integra o ciclo hidrológico (Lima, 2010). Os altos teores do sulfato podem refletir a oxidação de sulfetos (Figura 4), como a pirita, disseminados nos calcários e dolomitos do Grupo Bambuí (Misi et al., 2000).

Os teores do nitrato que excederam o limite de potabilidade foram relacionados a vulnerabilidade natural do aquífero cárstico à poluição, percolado por águas de infiltração, decorrente do uso do solo e desafios ao saneamento básico. A ingestão de águas com excesso de nitrato representa risco de agravos à saúde (Baird \& Cann, 2011), como a metahemoglobina ou síndrome do bebê azul, ligado a asfixia em recém-nascidos e câncer de estômago ou esôfago em adultos.

O teor ótimo de flúor (C), em função da temperatura média do ar local ( $\mathrm{T})$, foi obtido de acordo com Galagan \& Vermillion (1957) (Equações 1-2). Obteve-se um valor ótimo de fluoreto de $0,78 \mathrm{mg} . \mathrm{L}^{-1}$ para o Aquífero Bambuí, oeste da Bahia.

$$
\begin{aligned}
& €(T)=10,3+0,725 T \\
& C=22,2 / €
\end{aligned}
$$

Foi observada que 30 poços (25\%) excederam o limite de potabilidade local do flúor, de $0,8 \mathrm{mg} . \mathrm{L}^{-1}$, calculado de acordo com as Portarias 635/BSB (BRASIL, 1975) e 1469/00 (BRASIL, 2000). Segundo Mendes \& Oliveira (2004), o limite ótimo do flúor na água potável é função da temperatura média do ar local. Exige-se o monitoramento e vigilância em saúde ambiental do flúor no Aquífero Bambuí.
As concentrações do fluoreto no Aquífero Bambuí mantiveram-se sazonal e temporalmente similares (1970-2012) (Figuras 4-5), sugerindo uma origem natural, havendo valores extremos, outliers e tóxicos nos dados de campo e do SIAGAS. Segundo Misi et al. (2000); Misi et al. (2011), a fluorita ocorre disseminada nos calcários e dolomitos do Grupo Bambuí, na província cárstica do oeste da Bahia. No sítio de estudo, desconhece-se a adição sistemática de flúor na água de consumo humano pelo Sistema Autônomo de Água e Esgoto (SAAE), ou pela Empresa Baiana de Águas e Esgotos (EMBASA), como medida de saúde pública.

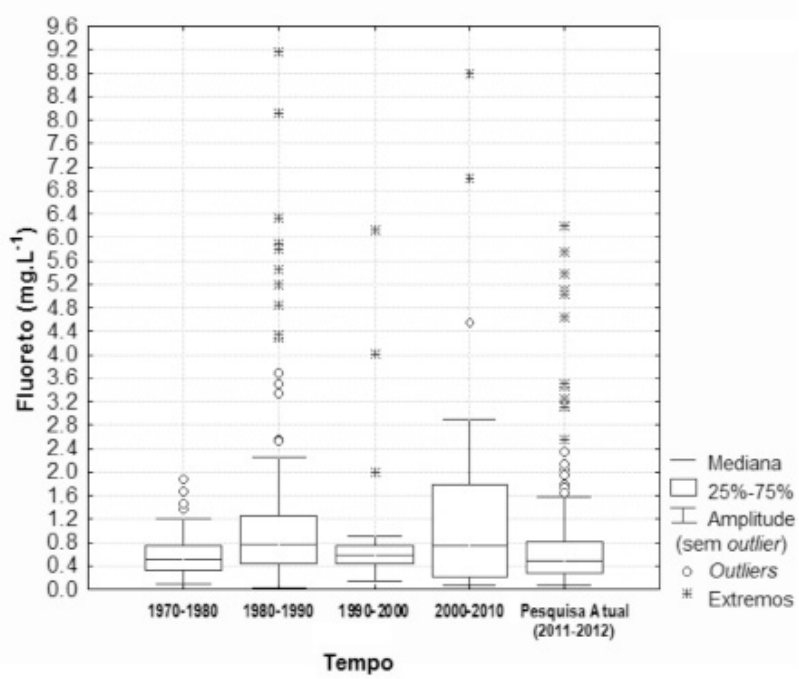

Figura 5 - Variabilidade temporal do flúor nas águas subterrâneas do sítio de estudo.

Velásquez et al. (2006) obtiveram prevalência de fluorose dentária de $81-97 \%, 30 \%$ na forma severa, entre 6-22 anos de idade, a qual foi atribuída ao consumo de doses naturais e tóxicas de flúor na água do Aquífero Bambuí, no norte de Minas Gerais. O consumo de água subterrânea rica em flúor no abastecimento é um fator de risco de fluorose dentária (Cangussu et al., 2004).

Cruz et al (2015) encontraram uma prevalência de fluorose dentária de $48,5 \%, 16,5 \%$ moderada/ severa, em escolares aos 12 anos (Índice de Dean), em Santana, na Bahia, relacionada à ingestão de água do Aquífero Bambuí. Estes resultados contrastam com a prevalência de fluorose, aos 12 anos, o Índice de Dean, de $17 \%$, severidade de $1,5 \%$ moderada ou severa, obtidas pelo levantamento de saúde bucal do Projeto SB Brasil 2010 (BRASIL, 2010).

A Figura 6 mostra uma espacialização dos teores de flúor nas amostras de água subterrânea, em que 4 poços no Setor Oeste (Coribe e São Félix do Coribe), 9 poços no Setor Leste (Carinhanha e Serra do Ramalho) e 13 poços em Santana superam o limite ótimo. As amostras de Santana excederam, 
localmente, o intervalo de teores médios de flúor na água subterrânea no mundo, de 0,1 a 3,0 mg.L-1, segundo Hem (1985); Edmunds \& Smedley (2004).

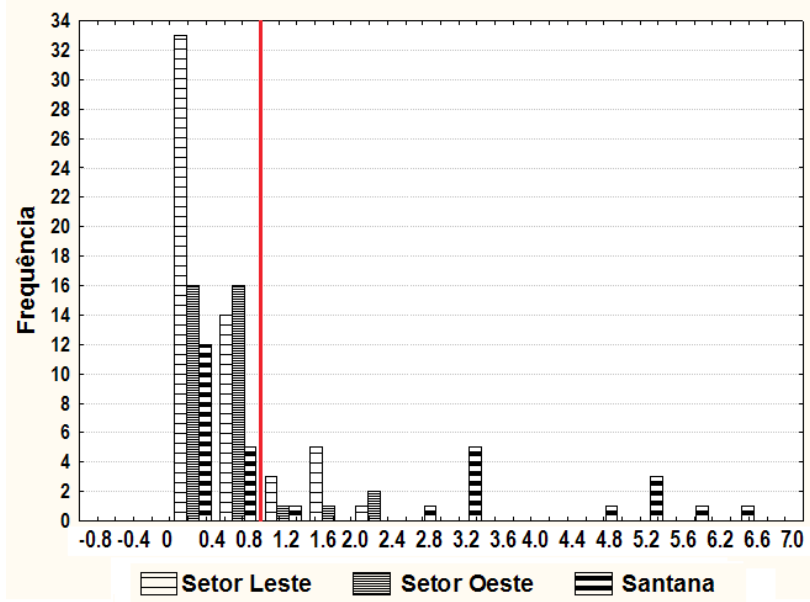

Figura 6 - Distribuição espacial dos teores de flúor nas amostras de água subterrânea.

Os níveis tóxicos de flúor em $21 \%$ das amostras, e $28 \%$ dos dados do SIAGAS, têm relevância epidemiológica, quanto a fluorose endêmica, segundo WHO (2006); Maheshwari (2006) (Tabela 3). A prevalência e a severidade da fluorose endêmica do tipo dentária, ou óssea estão relacionadas a ingestão prolongada de águas subterrâneas ricas em flúor (Boyle \& Chagnon, 1995; Wang \& Huang, 1995; Molina-Frechero et al., 2006; Tamer et al., 2007).

\section{Análise de componentes principais}

A técnica de análise das Componentes Principais (PCA), abrangeu 13 variáveis, em que quatro componentes descrevem $81 \%$ da variância total (Tabela 4). Tem-se a variabilidade do fluoreto explicada pelas componentes PC1 e PC2.

As componentes PC1 e PC2 descrevem $74 \%$ da variabilidade, definidas pelas variáveis $\mathrm{pH}, \mathrm{ORP}, \mathrm{STD}$, $\mathrm{DT}, \mathrm{Na}^{+}, \mathrm{K}^{+}, \mathrm{Ca}^{2+}, \mathrm{Mg}^{2+}$, $\mathrm{Cl}^{-}$e F- (Tabela 4). Refletem a ação do intemperismo químico e lixiviação de minerais, como a calcita, dolomita e a fluorita, constituintes das litofácies encaixantes do Aquífero Bambuí. Mostram a relevância do cloreto à salinização da água deste aquífero, que decorre da ação de fatores como as chuvas, evapotranspiração e a interação água-rocha.

As componentes PC1 e PC2 sugerem haver um paralelismo entre as variáveis $\mathrm{pH}, \mathrm{Na}^{+}$e $\mathrm{F}^{-}$, indicando que o flúor, principalmente como íons fluoreto, correlaciona-se negativamente com as variáveis $\mathrm{Ca}^{2+}, \mathrm{Mg}^{2+}$ e DT (de carbonatos). A solubilidade e precipitação da fluorita são controladas pelos teores do cálcio nas águas naturais (Equação 3), que segundo Yidana et al. (2012), a constante de equilíbrio $(K)$ da fase mineral fluorita, a temperatura de $25^{\circ} \mathrm{C}, \mathrm{K}_{\mathrm{S}}$, de $10^{-10,6}$.

$$
\mathrm{CaF}_{2} \leftrightarrow \mathrm{Ca}^{2}++2 \mathrm{~F}^{-}
$$

$\mathrm{Na}$ solução as atividades dos íons $\mathrm{Ca}^{2+}, \mathrm{F}^{-} \mathrm{e}$ $\mathrm{HCO}_{3^{-}}-\mathrm{CO}_{3}^{-2}$ são interdependentes (Handa, 1975; Rao, 2011) (Equações 3-5). Desta forma, por efeito de íon comum, e abundância do $\mathrm{Ca}^{2+}$ na água, de acordo com Apambire et al. (1997), a saturação da solução em calcita controla a solubilidade da fluorita.

A dissolução da calcita e dolomita das rochas do Grupo Bambuí, pelo $\mathrm{CO}^{2}$, fornece $\mathrm{Ca}^{2+}$ e $\mathrm{Mg}^{2+}$ à água (Equações 4-5). Entretanto, em pH alcalino predomina a reação inversa, que precipita a calcita (Hypolito et al., 2011). Pode ocorrer a dissolução da fluorita mesmo em solução saturada em calcita, sob influência de altas temperaturas do ar e pH alcalino, precipitando $\mathrm{Ca}^{2+}$ e $\mathrm{HCO}_{3}$.

$$
\begin{aligned}
& \mathrm{CaCO}_{3}+\mathrm{CO}_{2}+\mathrm{H} 2 \mathrm{O} \leftrightarrow \mathrm{Ca}^{2+}+ \\
& 2 \mathrm{HCO}_{3-} \text { (Eq. 4) }
\end{aligned}
$$

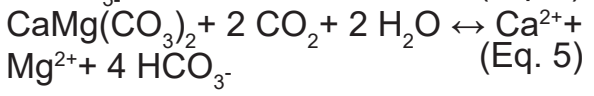

Handa (1975) propôs um modelo teórico para compreender a hidrogeoquímica do flúor, que pode ser sintetizado na Equação 6, em que $\mathrm{K}$ e a são respectivamente a constante de solubilidade e a atividade iônica. Este modelo elucida a correlação entre as os

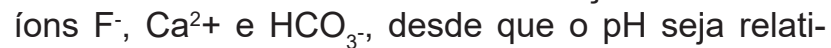
vamente constante. As reações de troca de base e a precipitação da calcita acrescentam íons $\mathrm{HCO}_{3}$ - à

\begin{tabular}{|c|c|c|c|c|c|c|c|c|c|c|}
\hline \multirow{3}{*}{ Parâmietros } & \multicolumn{5}{|c|}{ ATUAL } & \multicolumn{5}{|c|}{ SLAGAS } \\
\hline & \multirow{2}{*}{$\begin{array}{c}\begin{array}{c}\text { Risco de } \\
\text { Cárie }\end{array} \\
0.0-0.5\end{array}$} & \multirow{2}{*}{$\begin{array}{c}\text { Limite } \\
\text { ótimo } \\
0.5-1.0\end{array}$} & \multicolumn{2}{|c|}{$\begin{array}{c}\text { Fluorose } \\
\text { Dental }\end{array}$} & \multirow{2}{*}{$\begin{array}{c}\text { Fluorose } \\
\text { Óssea } \\
>\mathbf{3 . 0}\end{array}$} & \multirow{2}{*}{$\begin{array}{c}\begin{array}{c}\text { Risco de } \\
\text { Cárie }\end{array} \\
0.0-0.5\end{array}$} & \multirow{2}{*}{$\begin{array}{c}\text { Limite } \\
\text { Ótimo } \\
0.5-1.0\end{array}$} & \multicolumn{2}{|c|}{$\begin{array}{c}\text { Fluorose } \\
\text { Dental }\end{array}$} & \multirow{2}{*}{$\begin{array}{c}\begin{array}{c}\text { Fluorose } \\
\text { Óssea }\end{array} \\
>\mathbf{3 . 0}\end{array}$} \\
\hline & & & $1.0-1.5$ & $1.5-3.0$ & & & & $1.0-1.5$ & $1.5-3.0$ & \\
\hline Tamar & $61(50 \%)$ & $35(29 \%)$ & $5(4 \%)$ & $10(8 \%)$ & $11(9 \%)$ & $81(37 \%)$ & $77(35 \%)$ & $21(10 \%)$ & $19(9 \%)$ & $19(9 \%)$ \\
\hline Mín.- Máx. & $0.08-0.48$ & $0.51-0.94$ & 1.06 .1 .45 & $1.51-2.55$ & $3.11-6.2$ & $0.02-0.5$ & $0.50-0.98$ & $1.02-1.5$ & $1.68-2.9$ & $3.35-9.17$ \\
\hline Mediana & 0.27 & 0.64 & 1.11 & 1.85 & 4.65 & 0.33 & 0.73 & 1.20 & $2.1 \otimes$ & 5.45 \\
\hline Média $\pm D P$ & $0.28 \pm 0.01$ & $0.65 \pm 0.02$ & $1.17 \pm 0.07$ & $1.92 \pm 0.11$ & $4.42 \pm 0.35$ & $0.30 \pm 0.02$ & $0.71 \pm 0.02$ & $1.20 \pm 0.0 B$ & $2.20 \pm 0.0 \otimes$ & $5.60 \pm 0.40$ \\
\hline CV (\%) & 34.19 & 19.48 & 13.74 & 17.95 & 26.13 & 46.11 & 20.56 & 11.12 & 16.23 & 30.78 \\
\hline
\end{tabular}
solução, e elevam a solubilidade da fluorita.

Tabela 3 - Relação entre os teores de flúor nas águas naturais e fluorose endêmica. 
Tabela 4 - Matriz de correlação entre as componentes principais e as variáveis hidroquímicas.

\begin{tabular}{|c|c|c|c|c|c|c|c|c|}
\hline \multirow{2}{*}{ Varäveis } & \multicolumn{4}{|c|}{ Cherroso (2011) } & \multicolumn{4}{|c|}{ Seco(0012 } \\
\hline & PC1 & PQ2 & PG3 & PC4 & PC1 & PQ2 & PC3 & PCA \\
\hline $\mathrm{pH}$ & $\mathbf{0 . 5 5}$ & -0.43 & -0.09 & 024 & 0.71 & 027 & 026 & 0.09 \\
\hline ORP & 0.66 & 0.15 & 024 & 0.17 & 0.63 & 039 & 0.12 & 027 \\
\hline STD & 0.41 & 0.80 & 0.19 & 0.07 & 0.40 & 0.87 & 0.15 & 0.10 \\
\hline $\mathrm{Na}^{+}$ & 0.93 & 023 & 0.02 & -0.05 & $\mathbf{0 . 5 5}$ & $0 . \pi$ & 009 & 0.02 \\
\hline $\mathrm{K}^{+}$ & 0.45 & 0.52 & 027 & 0.45 & 001 & 0.72 & 0.10 & 0.44 \\
\hline $\mathrm{Ca}^{2+}$ & 0.74 & 0.59 & 006 & 0.17 & 0.93 & 0013 & 0.10 & 024 \\
\hline$M y^{2+}$ & 0.41 & 0.48 & 0.54 & 020 & 0.67 & 0.16 & 0.38 & 028 \\
\hline 0 & 004 & 0.81 & 0.44 & 006 & 0.40 & 0.73 & 0.43 & 003 \\
\hline $\mathrm{HOOS}$ & 0.14 & 0.43 & 0.44 & 0.66 & 025 & 0,30 & 0.61 & 0.57 \\
\hline $502^{2}$ & 0.16 & 0.40 & 0.52 & 0.59 & 0.47 & 032 & -0.42 & 0.50 \\
\hline Not & 0.31 & 028 & 0.68 & 026 & 026 & 000 & 0.74 & 002 \\
\hline $\mathrm{F}$ & 0.92 & 0.16 & 001 & 007 & 0.63 & 0.68 & 0.11 & 0.14 \\
\hline DT & 0.74 & 0.63 & 007 & 0.10 & 0.96 & 000 & 001 & 0.15 \\
\hline Varî̀nç Total $Q A$ & 312 & 293 & 116 & 896 & 332 & 290 & 11.4 & 75 \\
\hline Adumulada $P A$ & 312 & 605 & 72.1 & 81.1 & 332 & 622 & 73.7 & 81.1 \\
\hline
\end{tabular}

* Valores estatisticamente significativos representados em negrito.

(Eq. 6)

$$
\mathrm{KCaF}_{2} \leftrightarrow \mathrm{CaCO}_{3}=\left[\mathrm{a}\left(\mathrm{HCO}_{3}-\right)\right] /\left[\mathrm{a}\left(\mathrm{H}^{+}\right) \cdot\left(\mathrm{F}^{-}\right)^{2}\right]
$$

As componentes PC3 e PC4, abrangem 21\% da variância total, quais são descritas pelas variáveis $\mathrm{HCO}_{3}$, $\mathrm{SO}_{4^{-2}}$ e $\mathrm{NO}_{3}$ - (Tabela 4). A diferença entre os teores do $\mathrm{HCO}_{3}$ - nos períodos amostrais, condição não verificada para o $\mathrm{pH}$, foi explicada a uma provável adição na alcalinidade decorrente da ação de reações de troca de bases, as quais atuariam junto a coprecipitação dos íons $\mathrm{Ca}^{2+}$ e $\mathrm{HCO}_{3}$, no período de estiagem, contribuindo para a manutenção do $\mathrm{pH}$.

A geoquímica do flúor descrito para o Aquífero Bambuí, no oeste da Bahia, segue o modelo de Handa (1975) e o comportamento descrito por Apambire et al. (1997), Chae et al. (2007), Rafique et al. (2008), Rao (2011), Hu et al. (2013) e Vikas et al. (2013), em aquíferos cristalinos na Coreia, China, Índia e no Paquistão. Gonçalves (2014) demonstrou que o modelo de Handa (1975) e Rao (2011), pode ser aplicado, após adaptado, para hidrogeoquímica do flúor no Aquífero Bambuí.
Diniz (2006) e Costa (2011) sugeriram que o intemperismo e a lixiviação de plagioclásios adicionariam sódio à água subterrânea, no norte de Minas Gerais. As reações de troca de bases retirariam $\mathrm{Ca}^{2+}$ e acrescentariam íons de $\mathrm{Na}^{+}$litogênico à solução, aumentando o pH da solução e a solubilidade da fluorita. Explicaria a correlação negativa entre íons $\mathrm{Ca}^{2+}$ e $\mathrm{Na}^{+}$, no Aquífero Bambuí (BA).

Os minerais de argila (M), após a alteração de carbonatos impuros e pelitos do Grupo Bambuí, por meio das reações troca de bases (Equações 7-8), removem $\mathrm{Ca}^{2+}$ e fornecem íons de $\mathrm{Na}^{+}$e $\mathrm{HCO}_{3}$ - à solução, na reação direta.

$$
\begin{aligned}
& \text { (M) } \mathrm{SiO}_{3}+\mathrm{CO}_{2}+\mathrm{H} 2 \mathrm{O} \leftrightarrow \mathrm{M}_{2^{+}}+\mathrm{SiO} 2+ \\
& 2 \mathrm{HCO}^{3^{-3}} \\
& \mathrm{Na}(\mathrm{M})_{(\mathrm{S})}+\mathrm{Ca}^{+2} \leftrightarrow \mathrm{Ca}(\mathrm{M})_{(\mathrm{S})}+ \\
& 2 \mathrm{Na}^{+}
\end{aligned}
$$

A atividade do $\mathrm{Na}^{+}$precipita a calcita e permite o aumento da alcalinidade e solubilidade da fluorita 
(Apambire et al., 1997; Rao, 2011), visto nas Equações 8-9.

$$
\begin{aligned}
& \mathrm{CaF} 2+\mathrm{Na} 2 \mathrm{CO} 3 \leftrightarrow \mathrm{CaCO} 3+2 \mathrm{~F}-+ \\
& 2 \mathrm{Na}+ \\
& \mathrm{CaF}_{2}+2 \mathrm{NaHCO}_{3} \leftrightarrow \mathrm{CaCO}_{3}+2 \mathrm{Na}^{+}+ \\
& 2 \mathrm{~F}^{-} \mathrm{H}_{2} \mathrm{O}+\mathrm{CO}_{2}
\end{aligned}
$$

A análise multivariada sugere que a mineralização decorre da interação de fatores diversos, a exemplo das chuvas, evapotranspiração, interação água-rocha e circulação da água no aquífero. Também, que não pode ser observada correlação entre o nitrato e as demais variáveis hidrogeoquímicas investigadas.

Sugere-se que, além da interação água-rocha, as chuvas forneceriam íons $\mathrm{HCO}_{3}$ - para o aquífero e que o intemperismo de silicatos disponibilizaria sódio litogênico adicional à solução. A análise multivariada suportou a hipótese de haver um enriquecimento natural da água em flúor, porque a dissolução da fluorita depende da saturação desta em calcita e teores dos íons $\mathrm{HCO}_{3}$ e $\mathrm{Na}^{+}$.

\section{CONCLUSÕES}

Predominaram nas amostras águas alcalinas $(97 \%)$, sendo as fáceis bicarbonatadas cálcicas ou mistas cálcicas $(76 \%)$ as mais representativas. Ocorreram restrições de potabilidade locais quanto aos teores dos íons $\mathrm{Ca}^{2+}(71 \%), \mathrm{Na}^{+}(3 \%), \mathrm{Cl}^{-}(8 \%)$, $\mathrm{F}^{-}(25 \%), \mathrm{NO}_{3^{-}}(8 \%)$, STD $(5 \%)$ e DT $(7 \%)$.

No município de Serra do Ramalho, a poluição da água por nitrato deve ser monitorada, e identificada as fontes, merecendo a atenção das instituições responsáveis pelo saneamento básico e gestão da qualidade da água potável.

As análises hidrogeoquímica e multivariada sugerem haver dois aquíferos, um meio cárstico que abrange as fácies bicarbonatadas ou mistas cálcicas, e baixos teores de flúor, e outro pelito-carbonático representado pelas fácies bicarbonatadas sódicas e teores do flúor superior ao limite ótimo (25\%).

Propõe-se haver um controle hidrogeoquímico da distribuição dos teores de flúor na água subterrânea, desde que condições alcalinas e de altos teores de bicarbonato e sódio favoreçam a dissolução da fluorita, encontrada disseminada nas rochas do Grupo Bambuí, e a mobilidade do flúor na solução.

Esta pesquisa demonstrou relevância científica e social à elucidação da distribuição das doses tóxicas de flúor e à prevenção da fluorose dentária e óssea. Emerge a relevância do planejamento de ações integradas de gestão dos recursos hídricos, dessalinização de águas salobras, monitoramento da qualidade da água, educação e vigilância epidemiológica e de saúde ambiental.

\section{AGRADECIMENTOS}

Agradece-se em especial ao Conselho Nacional de Desenvolvimento Científico e Tecnológico (CNPq) e ao Grupo de Pesquisa Geoquímica das Interfaces (UFBA). Este trabalho está vinculado ao Projeto Hidrogeoquímica dos Aquíferos Cársticos de Irecê e Serra do Ramalho, Bahia, aprovado no Edital Universal do CNPq n.14/2011-Faixa B.

\section{REFERÊNCIAS}

Apambire, W.B.; Boyle D.R. \& Michel F.A. 1997. Geochemistry, genesis, and health implications of fluoriferous groundwaters in the upper Regions of Ghana. Environmental Geology. 33 (1): 13-24.

Boyle, D. R; Chagnon, M. 1995. An incidence of skeletal fluorosis associated with groundwaters of the Maritime Carboniferous Basin, Gaspe Region, Quebec, Canada. Environ Geochem Health. 17: $5-12$.

Baird, C. \& Cann, M. 2011. Química Ambiental. $4^{\mathrm{a}}$ Edição. Bookman, Porto Alegre, 844p.

Bell, F.G. 1998. Environmental Geology. Principies and Practice. Blackwell Science, London, 594 p.

Brasil. Ministério da Saúde. Secretaria de Atenção à Saúde. Departamento de Atenção Básica. Coordenação Nacional de Saúde Bucal. Projeto SB Brasil 2010 - Condições de saúde bucal da população brasileira 2010: resultados principais. Brasília: MS-CNSB, 2010.

Brasil. Ministério da Saúde. Portaria 635 de 26 de dezembro de 1975. Aprova normas e padrões sobre a fluoretação da água de sistemas públicos de abastecimento. Brasília, 19 jan 2001. (Seção 1.p.18-22).

Brasil. Ministério da Saúde. Portaria 1.469, de 29 dez. 2000. Estabelece os procedimentos e responsabilidades relativos ao controle e vigilância da qualidade da água para consumo humano e seu padrão de potabilidade, e dá outras providências. Diário Oficial da União, 2001.

Brasil. Ministério da Saúde. Portaria 2914 de 12 de Dezembro de 2011. Dispõe sobre os procedimentos de controle e de vigilância da qualidade da água para consumo humano e seu padrão de potabilidade. Diário Oficial da União, 2011.

Brindha, K.; Rajesh, R.; Murugan, R. \& Elango, L. 2011. Fluoride contamination in groundwater in parts of Naglonda, Andhra Pradesch, India. Environ Monit Assess. 172 (1-4): 481- 492. 
Camurugy, E.B. 2009. Uso da hidroquímica e isótopos estáveis ( $\delta \mathrm{D}$ e $\delta 180$ ) no estudo da qualidade e origem das águas subterrâneas do aquífero cárstico da região oeste do Estado da Bahia. Monografia de Graduação. Universidade Federal da Bahia. Instituto de Geociências. 66p.

Cangussu, M.C.T.; Fernandez, R.A.C.; Rivas, C.C.; Ferreira Jr., C. \& Santos, L.C.S. 2004. Prevalência da fluorose dentária em escolares de 12 e 15 anos de idade em Salvador, Bahia, Brasil, 2001. Caderno de Saúde Pública, Rio de Janeiro. 20 (1): 129-135.

Chae, C.T.; Yun, S.T.; Mayer, B.; Kim, K.H.; Kim, S.Y.; Kwon, J.S.; Kim, K. \& Koh, Y.K. 2007. Fluorine geochemistry in bedrock groundwater of South Korea. Science of the Total Environment. 385 (13): $272-283$.

CODEVASF. Companhia de Desenvolvimento do Vale do São Francisco. 1989. Inventário Hidrogeológico da Serra do Ramalho. v.1, Brasília, 84 p.

Costa, D. A. 2011. Controle lito-estrutural e estratigráfico na hidrogeoquímica e nas concentrações de fluoreto no Sistema Aquífero Cárstico - Fissural do Grupo Bambuí, norte de Minas Gerais. Dissertação de Mestrado. Universidade Federal de Minas Gerais. Instituto de Geociências. 131p.

Costa, S. M.; Abreu, M.H.N.G.; Vargas, A.M.D.; Vasconcelos, M.; Ferreira, F.E. \& Castilho, L.S. de. 2013. Dental caries and endemic dental fluorosis in rural communities Minas Gerais, Brazil. Revista Brasileira de Epidemiologia.16 (4): 1021-1028.

Coutinho, C.A.M. 2014. A fluorose dentária na região cárstica do município de Santana-BA: definição de áreas de risco para consumo humano das águas subterrâneas com base nos dados hidroquímicos e epidemiológicos. Dissertação de Mestrado. Universidade Federal da Bahia. Instituto de Geociências. 164p.

Cruz, M.J.M., Coutinho, C.A.M. \& Gonçalves, M.V.P. 2015. The Dental fluorosis on Santana karst region, Bahia State, Brazil. Journal of Geography. 3(2): 51-67.

Dardenne, M.A., Ronchi, L..H., Bastos Neto, A.C. \& Touray, J.C. 1997. Geologia da fluorita. Principais depósitos minerais do Brasil. DNPM/CPRM, v4, 479-508p.

Diniz, I. G. 2006. O flúor nas águas subterrâneas do estado de minas gerais. Dissertação de Mestrado. Universidade Federal de Minas Gerais. Instituto de Geociências. 163p.

Drever, J.I. (Ed.). 1997. The Geochemistry of natural waters: surface and groundwater environments. Prentice-Hall, New Jersy, p. 436.

Edmunds, M.; Smedley P. 2005. Fluoride in natural waters. In: Selinus, O.; Alloway, B.; Centeno, J. A;
Finkelman, R. B.; Fuge, R.; Lindh, U.; Singh, H.; Smedley, P. (Eds.). Essentials of medical geology: impact of the natural environment public health. Elsevier Academic Press, Amsterdã. p 301-328.

Fairchild, I.J.; Borsato, A.; Tooth, A.F.; Frisia, S.; Hawkesworth, C.J.; Huang, Y.; Mcdermott, F. \& Spiro, B.F. 2000. Controls on trace element $\mathrm{Sr}-\mathrm{Mg}$ compositions of carbonate cave waters: implications for speleothem climatic records. Chemical Geology. 166: 255-269.

Ferreira, E.F.; Vargas, A.M.D., Castilho, L.S.; Velásquez, L.N.M.; Fantinel, L.M. \& Abreu, M.H.N.G. 2010. Factors Associated to Endemic Dental Fluorosis in Brazilian Rural Communities. Int J Environ Res Public Health. 7 (8): 3115-28.

Fujibayashi, S.Y., Archetti, F.B., Pizzatto, S., Losso, E.M. \& Pizzatto, E. 2011. Severidade de fluorose dental em um grupo de Escolares. RSBO. 8 (2): 168-73.

Galagan, D.J \& Vermillion, J.R. 1957. Determining optimum fluoride concentrations. Public Health Rep. U.S. 72: 491-93.

Gallará, R. V.; Piazza, L.A; Piñas, M.E.; Barteik, E.; Moncunill, I. \& Ponce, R.H. 2011. Fluorosis endémica en zonas rurales del norte y noreste de la provincia de Cordoba, Argentina. Revista de Salud Pública. 15 (1): 40-48.

Gonçalves, M.V.P. 2014. Flúor nas águas subterrâneas do Aquífero Bambuí no sudoeste da Bahia (Brasil). Tese de Doutorado. Universidade Federal da Bahia. Instituto de Geociências. 193p.

Handa, B.K. 1975. Geochemistry and genesis of fluoride-containing ground waters in India. Ground Water. 13 (3): 275-280.

Hem, J.D. 1985. Study and interpretation of the chemical characteristics of natural water. U.S. Geological Survey Water-Supply Paper. (2254):1-263.

Hypólito, R; Ezaki, S. \& Andrade, S. 2011. Geoquímica da Interação Água/Rocha/Solo: Estudos Preliminares. $1^{\text {a }}$ Edição. ALL PRINT, São Paulo, $454 \mathrm{p}$.

Hu, S.; Ting, L. \& Jing, C. 2013. Principal component analysis of fluoride geochemistry of groundwater in Shanxi and Inner Mongolia, China. 2013. Journal of Geochemical Exploration, v. 135: 124 - 129.

IBGE. Instituto Brasileiro de Geografia e Estatística. 2010. Dados do Censo demográfico 2010 publicados no Diário Oficial da União do dia 24/11/2010.

INMET. Instituto Nacional de Meteorologia. Balanço hídrico e dados climatológicos. Disponível em: http://www.inmet.gov.br/html/agro.html. Acesso em 11.10.2013. 
Komati, S.H. \& Figueiredo, B.R. 2013. Flúor em água e prevalência de fluorose em Amparo (SP). Geociências (São Paulo). 32 (3): 547-559.

Krauskopf, K. \& Bird, D. 1995. Surface chemistry: the solution-mineral interface. Introduction to geochemistry (Ed MG-HI Editions) Mc Graw-Hill International Editions edn. Earth Sciences and Geology Series, 135-163 p.

Licht, O.A.B.; Morita, M.C. \& Tarvainen, T. 1996. A utilização de dados de prospecção geoquímica da fluorita no primeiro planalto paranaense na identificação de áreas de interesse para a saúde pública: Uma abordagem preliminar. Geochimica Brasiliensis. 10 (1): 57-69.

Lima, A.S. 2010. Composição e origem das águas minerais naturais: exemplo de Caldas da Saúde. Almedina, Coimbra, 246p.

Logan, J. 1965. Interpretação de análises químicas da água. U.S. Agency for International Development, Recife, 67p.

Maheshwari, R. C. 2006. Fluoride in Drinking Water and its Removal. Journal of Hazardous Materials. 137: 456-463.

Mendes, B. \& Oliveira, J.F.S. 2004. Qualidade da água para o consumo humano. Lidel, Edições Técnicas, Lisboa, 640p.

Misi, A.;lyer, S.S.; Coelho, C.E.S.;Tassinari, C.C.G.;Rocha, W.J.S.F.;Gomes, A.S.R.; Cunha, I.A.;Toulkeridis, T.\& Sanches, A.L. 2000. A metalogenic evolution model for the lead-zinc deposits of the Meso and Neoproterozoic sedimentary basins of the São Francisco Cráton, Bahia and Minas Gerais, Brazil. Revista Brasileira de Geociências. 30 (2): 302-305.

Misi, A.; Kaufman, A.J.; Azmy, K.; Dardenne, M.A.; Sial, A.N. \& de Oliveira, T.F. 2011. Chapter 48 Neoproterozoic successions of the Sao Francisco Craton, Brazil: the Bambui, Una, Vazante and Vaza Barris/Miaba groups and their glaciogenic deposits. Geological Society. Memoirs (London). 36: 509-522.

Molina-Frechero, N.; Castañeda-Castaneira, E., Bologna-Molina, R. \& Carlos, J. 2006. Fluorosis endémica en una población asentada a la altitud de 2,100 m. Pediatría. 73 (5): 220-214.

Moral, F.; Cruz-Sanjulián, J.J. \& Olías, M. 2008. Geochemical evolution of groundwater in the carbonate aquifers of Sierra de Segura (Betic Cordillera, southern Spain). Journal of Hydrology. 360 (1-4): 281-296.

Naseem, S.; Rafique, T.; Bahir, E.; Bhanger, M.I.; Laghari, A. \& Usmani, T.H. 2010. Lithological influences on occurrence of high-fluoride groundwater in Nagar Parkar area, Thar Desert, Pakistan. Chemosphere. 78: 1313-1321.
Negrão, F.I. 2007. Hidrogeologia do Estado da Bahia: qualidade, potencialidade, disponibilidade, vulnerabilidade e grau de poluição. Tese de Doutorado. Instituto Universitario de Xeoloxía Isidro Parga Pondal. Universidade da Coruña. 195p.

Parreiras, P.M. de; Silva, A.P.A. \& Zocratto, K.B.F. 2009. Fluorose dentária: percepção dos portadores e seus responsáveis. Revista da Faculdade de Odontologia. 14 (1): 18-22.

Piper, A.M. 1944. A graphic procedure in the geochemical interpretation of water analyses. Trans Am Geophys Union, v.25, 914-923p.

PNUD. Programa das Nações Unidas para o Desenvolvimento. 2010. Ranking Decrescente do IDH-M dos Municípios do Brasil. Atlas do Desenvolvimento Humano no Brasil. Acesso em: jul.2017. Disponível em: <http://www.pnud.org. br/atlas/>.

Rao, S.N. 2011. High-fluoride groundwater. Environ Monit Assess. 176: 637-645.

Rafique, T.; Naseem, S.; Bhanger, M. I. \& Usmani, T. H. 2008. Fluoride Ion Contamination in the Groundwater of Mithi Sub-District, the Thar Desert, Pakistan. Environmental Geology. 56: 317-326.

Raju, N.J. 2017. Prevalence of fluorosis in the fluoride enriched groundwater in semi-arid parts of eastern India: Geochemistry and health implications. Quaternary International. 443: 265-278.

Rebouças, A.C. 2006. Água Doce no Mundo e no Brasil. In: Rebouças, A.C.; Braga, B. \&Tundisi, J. G (ORG). Águas Doces no Brasil: capital ecológico, uso e conservação. Escritura Editora, São Paulo. 1-35pp.

Rigo, L.; Sabadin, C.S.; Wietholter, P.; Solda, C.; \& Flores, R. A. 2014. Prevalência de fluorose dentária em crianças de uma escola municipal de Passo Fundo/RS. Full Dent. Sci .5 (19): 472-476.

Soares, F.F.; Valverde, L.F.; Silva, R.D.C.R. \& Cangussu, M.C.T. 2012. Prevalência e severidade de fluorose em escolares do município de São Francisco do Conde-BA, 2010. Rev Odontol UNESP. 41 (5): 318-323.

Tamer, M. N., Köroğlu, B. K., Arslan, Ç., Akdoğan, M., Köroğlu, M., Çam, H., \& Yildiz, M. 2007. Osteosclerosis due to endemic fluorosis. Science of the total environment. 373 (1): 43-48.

Toassi, R.F.C. \& Abegg, C. 2005. Fluorose dentária em escolares de um município da serra gaúcha, Rio Grande do Sul, Brasil. Cad. Saúde Públ., Rio de Janeiro. 21 (2): 652-655.

Velásquez, L.N.M.; Fantinel, L.M.; Castilho, L.S; Uhlein, A.; Vargas, A.M.D.; \& Aranha, P.R.A. 2006. Fluorose dentária e anomalias de flúor na água subterrânea no município de São Francisco, Minas 
Gerais. In: Silva, C. R. da; Figueiredo, B. R.; De Capitani, E. M.; Cunha, F. G. da. (Org). Geologia Médica no Brasil. Rio de Janeiro: CPRM - Serviço Geológico do Brasil. 6: 110-117 p.

Vikas, C.; Kushwaha, R.; Ahmad, W.; Prasannakumar, V. \& Reghunath, R. 2013. Genesis and geochemistry of high fluoride bearing groundwater from a semiarid terrain of NW India. Environmental Earth Science. 68: 289-305.

Yidana, S.; Banoeng-Yakubo, B.; Aliou, A. \& Akabzaa, T. 2012. Groundwater Quality in Some Voltaian and Birimian Aquifers in Northern Ghana - An Application of Multivariate Statistical Methods and Geographic Information Systems. Hydrological Sciences Journal. 57: 1168-1183.

Wang, L.F.; Huang, J. Z. 1995. Outline of control practice of endemic fluorosis in China. Soc Sci Med, 41: 1191-1195.

White, W, B. 2002. Karst hydrology: recente developments and open questions. Eng. Geol, 65: 85-105.

W.H.O. World Health Organization. 2006. Guidelines for drinking-water quality. $3^{a}$ Edição. v.1. Recommendations, Geneva. 